\title{
Case-based Approaches to Professional Ethics: a systematic comparison of students' and ethicists' moral reasoning
}

\author{
MATTHEW KEEFER
}

University of Missouri-St Louis, USA

\section{KEVIN D. ASHLEY}

University of Pittsburgh, USA

ABSTRACT This article provides a systematic analysis of the cognitive processes required for acquiring skill in practical ethical reasoning in a professional domain. We undertook this $N S F$-supported research project in part to study relationships between case-based instruction in professional ethics and cognitive analyses of ethical reasoning strategies. Using a web-based experimental design, we report striking differences in the students' and ethicists' use of knowledge and reasoning. Virtually all of the ethicists and some students' protocols made significant use of specialized professional knowledge and also used role-specific content in the ethical principles applied in their responses. In contrast, other student protocols made significantly more use of common knowledge and applied more general ethical principles or appealed to consequences in the justification of their responses. Our analyses show how certain strategies were superior to others in regard to identifying alternative moral issues, assessing the moral implications of actions, and providing alternative practical resolutions to conflicts. The findings point to the importance of professional knowledge and role-specific professional obligations in resolving professional ethical conflicts: the same knowledge and "middle-level" principles necessary to comprehend and apply professional codes of ethics.

In considering professional ethical reasoning from the viewpoint of cognitive science, an area of primary interest concerns students' abilities to recognise and respond appropriately to ethical problems characteristic of their professional practice. Increasingly, professional schools in higher education are including ethics courses in their programs and increasingly the pedagogical methods of these courses have relied on the use of case-based instructional strategies. The pedagogical value of focusing ethics instruction on individual cases, on case-based comparison, and on explicit procedures for conducting moral deliberation has been recognised by Jonsen and 
Toulmin (1990), Jonsen (1991), Griffin (1988) Arras (1991), Beauchamp and Childress (2000), Harris et al. (1999) and Whitbeck (1998).

One way that educators have attempted to alert young professionals to the responsibilities that attach to professional knowledge has been to stress the importance of codes of ethics. Ethics codes are not only a fact of life in professional practice. Codes of ethics provide moral principles aimed at guiding practice; their provisions apply the principles of common morality to a specific professional field by specifying additional, more domain-specific principles. These role-specific principles extend common morality by articulating the special obligations pertaining to professional roles.

Students need to have a working knowledge of the provisions of their code in order to align their ethical conduct to professional standards. Yet, while we know that knowledge of professional codes is important, many authors note that most professionals do not frequently consult ethics code provisions as a guide in making decisions (see Harris et al., 1999). This would suggest that much of the practical and ethical knowledge that is characteristic of a domain is acquired "on the job" (Resnick \& Wirt 1996; Lave \& Wenger, 1991).

From a cognitive viewpoint, applying ethical codes is difficult partly due to the open-ended quality of professional codes. For example, in the domain of engineering, numerous code provisions such as those involving "conflicts of interest" or admonitions to "recognize the proprietary interests of others", "hold paramount the safety, health, welfare, of the public in the performance of their professional duties" or "act in professional matters for each employer or client as faithful agents or trustees" involve deliberately open-textured terms which are not further defined in the codes (Harris et al., 1995, pp. 6-7). Hence, simple knowledge of code provisions is clearly not enough to help students learn to recognise ethical dilemmas they are likely to encounter in their professional practice.

Knowledge of these role-specific obligations along with relevant specialised knowledge is necessary if professionals are to identify ethical problems that they are likely to encounter in their professional life. We believe that students will only understand and be guided by ethics code provisions if they gain experience working through case examples and explanations that identify the problem features which code principles mark as ethically relevant. Specifically, we are interested in the way common morality is applied to professional contexts and understanding the role that specialised professional knowledge plays in the application of these domain-specific middle-level principles, e.g. those principles that might appear in professional ethics code provisions.

\section{Our Model of Case-based Moral Reasoning}

The model we have devised to analyse case-based moral reasoning is informed by our own previous work and uses techniques of discourse analysis, AI research in cognitive science, analogical reasoning and problem-solving (Ashley, 1990; Keefer \& Olsen, 1995; Ashley \& Keefer, 1996; Keefer et al., 1998). It is also closely aligned with new approaches to the teaching of practical ethics (e.g. Griffin, 1988; Arras, 
1991; Jonsen, 1991; Whitbeck, 1998), especially those found in a text such as Engineering Ethics: Concepts and Cases (Harris et al., 1999).

Our model and these recent philosophical approaches share a commitment to studying and teaching ethics using analyses of moral decision-making in practical contexts, usually in the form of realistic case examples. For example, professional ethics texts such as Engineering Ethics (Harris et al., 1995) provide many ethics problems and analyses invoking and interpreting general provisions of common morality and specific ethics code provisions.

The pedagogical efficacy of such an approach relies on the willingness of students to work through the numerous case examples and to compare cases in light of the ethicists' detailed case analyses. The case examples in Engineering Ethics are presented to students in an interesting way. Often, the authors organise a series of cases around a principle drawn from common morality such as "it is wrong to steal or to commit theft" or from an engineering ethics code provision such as "Engineers shall not disclose confidential information concerning the business affairs or technical processes of any present or former client or employer without his consent ([NPSE code] III.4)" (Harris et al., 1995, p. 126).

Our model has been devised to help us classify in a systematic way the analyses of such ethical problems as performed by engineering students or by ethicists. The model supports classification of four aspects of a case-based analysis: the components of the analysis, its complexity, the content of the moral justification in the analysis and the knowledge invoked in the analysis.

\section{Components in Case-based Analysis}

According to our model, one may observe seven basic components in case-based moral reasoning, as summarised in Table I. A reasoner may (1) identify the moral issue at stake as well as (2) the relevant known and unknown facts in the problem, (3) offer a resolution and (4) a moral justification, (5) consider alternative scenarios that argue for different conclusions, (6) identify and evaluate longer term moral consequences and (7) offer alternative resolutions. For example, according to our model, when we observe a subject's analysis identifying moral issues, we encode it as Component 1 . If the analysis also invokes some ethical principles as justifications, we encode it as Component 4 as well.

Components 5 and 7 of our model account for some specific aspects of the process of moral analysis described in (Harris et al., 1995, Figs 4.1, p. 102; 4.3, p. $111 ; 5.1$, p. $128 ; 5.2$, p. 140).

In our study, some subjects' case analyses proceeded in a decidedly casuist way. They presented a series of examples along a spectrum from clear positive to clear negative (e.g. the "line-drawing" method in Harris et al., 1995, p. 131). Each successive case represents an addition of or a change in the magnitude of some morally relevant factor or condition which makes the application of common or specialised middle-level moral principles more or less necessary (Harris, et al., 1995, p. 131). In our model we code this tactic as reasoning Component 5, generating alternative scenarios. 
TABLE I. Component model of moral case-based reasoning

\begin{tabular}{ll}
\hline Component 1 & Identify the moral issue at stake \\
Component 2 & Identify the relevant knowledge and unknown facts in a problem \\
Component 3 & Offer a resolution \\
Component 4 & Offer a moral justification \\
Component 5 & Consider alternative scenarios that argue for different conclusions \\
Component 6 & Identify and evaluate longer term moral consequences \\
Component 7 & Offer alternative resolutions using interactive planning \\
\hline
\end{tabular}

Other subjects organised case analyses around conflicting middle-level code principles, such as engineers should "hold paramount the safety, health, welfare, of the public in the performance of their professional duties" versus "act in professional matters for each employer or client as faithful agents or trustees" (Harris et al., 1995, p. 139). Subjects also annotated cases presented as calling for "hard choices" between conflicting principles with a range of optional actions the protagonist could take (referred to as creative middle-way solutions). The actions are organised by the extent to which they serve each of the conflicting principles (i.e. in a manner that satisfies all, or most, of the moral claims in the dilemma). In our model we classify this kind of reasoning as Component 7 , alternative actions. In previous work, the first author established a strong empirical correlation between protocols using these alternative action resolutions (involving interactive plans and social episodes) and protocols scored as exhibiting Carol Gilligan's Care Orientation to moral reasoning (Keefer \& Olson, 1995).

\section{Complexity of Case-based Analysis}

Based on our empirical investigations, described below, students often provide a basic case analysis comprising either a simple plan for resolving the problem (Component 3) or a small set of alternative resolutions (Component 7 ) as well as a moral justification, for instance, by invoking some ethical principles (Component 4). Other responses are more complex, and our model includes a scheme for classification of complexity of analyses as shown in Table II. The basic response of Component 3 and 4, or 7 and 4, are classified as Component Level A. If a subject includes not only a resolution but also a combination of two components the response is classified as Level $\mathrm{B}$. These may include a justification (Component 4), identification of a moral issue (Component 1), identification of known and unknown relevant facts (Component 2), identification of moral consequences (Component 6), or alternative scenarios where conclusions differ (Component 5). Level C involves a combination of three such elements, and four elements merit classification as Level D. 
TABLE II. Definition of component complexity levels

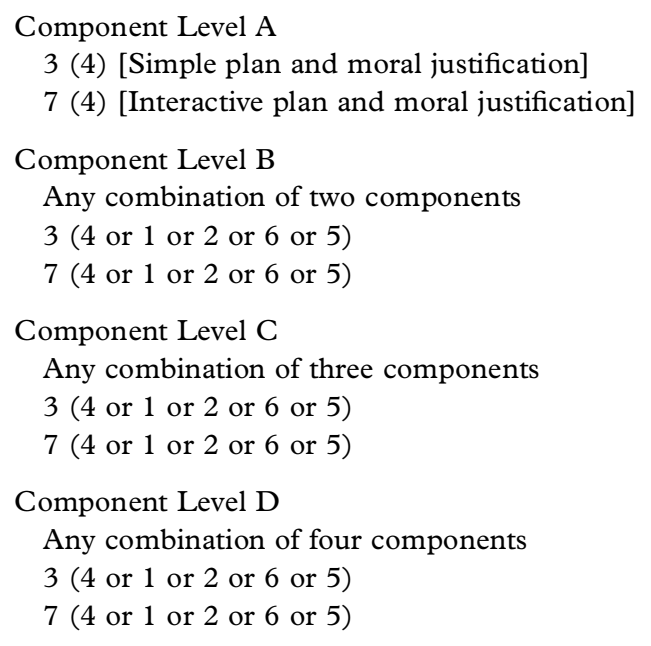

\section{Content of Moral fustification}

We have also developed a scheme, set out in Table III, for classifying the content of the moral issues or justifications subjects provide in Component 1 and 4 . According to this aspect of our model, when subjects (i.e. engineering students or ethicists) invoke middle-level principles in their analyses to specify responsibilities that attach to professional knowledge and training (e.g. ethics code provisions) we encode the content of these moral issues and justifications as role specific obligations or RSO. This classification may be contrasted to justifications made by appeal to simple consequences, CONS, or to more general middle-level principles such as a norm or rule PRINC (e.g. do not steal).

In our study of engineering students and ethicists, we have adopted a fourth content classification for Component 4 justifications: MT for moral theory. Chapter 6 of (Harris et al., 1995) discusses the use of moral tests in moral problem solving. An analysis based on moral tests appeals to higher-level principles derived from moral theories (e.g. deontology, utilitarianism, etc.) drawn from the field of philosophical ethics. These tests are useful, these authors suggest, to decide which side or "horn" of a dilemma is better justified when faced with cases requiring a hard choice between options.

The principles that these test or theories articulate derive directly from philosophical ethics and, so, represent a very high degree or abstract generality. For

TABLE III. Definition of content of moral justifications

$\begin{array}{ll}\text { PRINC } & \text { Justification by appeal to middle-level principle or norm } \\ \text { CONS } & \text { Justification by appeal to consequences } \\ \text { RSO } & \text { Justification by appeal to role-specific obligations } \\ \text { MT } & \text { Justification by appeal to moral theory }\end{array}$


TABLE IV. Component 2: identifying relevant knowledge

\begin{tabular}{ll}
\hline K (SK) & Specialised knowledge-relevant known facts \\
$\mathrm{U}(\mathrm{SK})$ & Specialised knowledge-relevant unknown facts \\
$\mathrm{H} / \mathrm{I}(\mathrm{SK})$ & Specialised knowledge-relevant hypothetical or inferred facts \\
$\mathrm{K}(\mathrm{CK})$ & Common knowledge-relevant known facts \\
$\mathrm{U}(\mathrm{CK})$ & Relevant unknown facts-common knowledge \\
$\mathrm{H} / \mathrm{I}(\mathrm{CK})$ & Common knowledge-relevant hypothetical or inferred facts \\
\hline
\end{tabular}

example, deontological moral principles rely on tests to determine whether one's reasons for action are categorical, prescriptive and universalisable in form, (e.g. treat others only as ends and never as means to ends). Such principles are highly abstract as they identify reasons that anyone ought to have independent of their own motives or character, professional role or context. Similarly, utilitarian strategies employ tests using highly abstract and general principles (e.g. weigh the value of options using a commensurate scale and maximise utility) that also can or ought to be applied by anyone in any moral context. In our study, we classified these principles and tests as MT, meaning justifications that appeal to moral theory.

It is important to note that among the middle-level principles, PRINC and CONS appear to be more general and less context-dependent than RSO. Indeed, we believe this to be the case and there is evidence to follow that will support this claim. However, we consider it important to code different levels of moral principles (middle and high) since it is a matter of some contention whether highly abstract moral principles (e.g. derived from moral theory) play a significant role in moral decision-making (Jonsen \& Toulmin, 1990; Walzer 1995).

\section{Knowledge Invoked in Case-based Analysis}

Finally, our model characterises the knowledge about a fact situation subjects invoke in their case-based analyses (Component 2) along two dimensions: (1) whether they involve specialised knowledge (e.g. technical engineering knowledge) or common knowledge and (2) whether the relevant facts are known, unknown, or hypothetical or inferred. Accordingly, relevant knowledge is broken down into the six subcategories that are summarized in Table IV.

\section{Development of our Model in an Earlier Study}

We developed our model in an earlier study, in which we compared written protocols of analyses of ethical cases prepared by graduates in an ethics programme and by high school students. The cases dealt with quasi-medical ethics, not engineering ethics. We noticed that the graduate students used moral analyses and heuristics that resembled the commentaries of experienced ethicists outlined in Engineering Ethics. Upon closer analysis we found that our Component Model, 
which we had derived independently after empirical study, closely matched the case commentaries and reasoning strategies explicitly recommended by the authors of Engineering Ethics.

In designing this initial experiment, our hypothesis had been that more experienced ethical reasoners would adopt a more explicitly "casuistic" reasoning strategy. We expected to see some evidence of explicit comparisons to cases along the lines of the methodology described in (Strong, 1988). We had, moreover, consciously built into the experiment an opportunity to compare and contrast cases. Our case examples were written with the aim of promoting explicit case comparison in the manner of the casuist or "line-drawing" approach outlined in Engineering Ethics. The dilemmas that were presented to subjects were selected so that subjects could have drawn analogies from one problem to another, either to show that they presented the same principles and/or role-specific obligations, the same conflicts among principles, or an interesting comparison of factual circumstances.

While explicit reference to earlier cases was rare, we found that many of the analyses and explanations that the graduate students used closely modelled those employed by the authors of Engineering Ethics in the following respects. For one thing, whereas the text's analysis might include a series of clear positive and negative examples (intentionally varied to obtain pedagogical goals), some of our subjects spontaneously generated the same strategies in order to explain or justify their resolutions of the dilemma.

To illustrate using one of our case examples from this study, a dilemma describes a 73-year-old woman with Alzheimer's disease. The woman's son tells the physician that he is no longer able to care for his mother (due to other responsibilities), and wishes his mother to enter a nursing home. The son further requests the physician not tell his mother about her condition "since it would only scare her". The question to the subject is whether or not the physician should honour the son's request.

It is not clear why the doctor is communicating with the son, and not the patient, in the first place. That issue aside, the doctor should tell his patient that she has Alzheimer's disease, unless he has reason to believe she is suicidal and will react badly to the information, or that she is demented so that she will not understand the information conveyed. Unless these extreme circumstances obtain, there is no reason to override the prevailing assumption that patients should be given accurate and complete information about their conditions. If the son is concerned about "scaring" her, he should realise that she is likely aware of her condition, and will realise upon being put in a nursing home that something is wrong, both of which are "scary" issues too. The patient should participate as much as possible in the planning about the nursing home.

This subject's strategy bears a close similarity to the example of a line-drawing or graduated response that is described in Engineering Ethics (Harris et al., 1995, p. 130). Here the subject establishes a negative paradigm for the purpose of a comparison that shows the (extreme) conditions or unknown facts, which would 
need to be known or in place to honour the son's wish and to override the physician's obligation to inform the patient of her condition. In the protocol analysis that was used in our earlier study, part of this response was coded as an alternate scenario (Component 5).

We also found other important parallels between the graduate students' responses and the pedagogical strategies promoted in Engineering Ethics. For example, virtually all of the graduate students were careful to specify issues and conditions (whether stated in the dilemma or inferred as hypothetical) which supported the application of principles or recommended actions. They also identified relevant information not in the dilemma but still deemed necessary or useful for arriving at a satisfactory practical resolution (as in the above example). We also found that the graduate students were better able to specify conditions under which specific professional role obligations, the kind of obligations described in professional ethics codes, recommend particular actions.

The following response is an example of specifying the conditions necessary for a paradigm/case to override the patient's autonomy. The response was to a case in which a physician, Diana, is considering whether or not to "deceive" her patient, whom she suspects is addicted to narcotics, by administering a placebo in place of her medication for pain. Note that most of the conditions specified presuppose some expert knowledge of special obligations of medical practice:

It may be that Diana is justified in "deceiving" her patient, provided that the following questions are affirmatively answered: (1) does Diana have sufficient reason to believe her patient is addicted; (2) is the patient's current dosage of painkillers sufficient for other patients in similar circumstances; (3) is the patient likely to recover so that addiction is undesirable for future life plans; (4) has Diana attempted to provide [refer the patient for] psychiatric, support group or pain relief care which is non-medicinal; (5) has Diana attempted to communicate her concerns directly to the patient?

In addition, our protocol analysis had also coded for responses that included creative middle-way solutions (Component 7), strategies also promoted in Engineering Ethics. We defined these as instances where subjects decided to generate a practical solution that satisfies all, or most of, the moral issues at risk in the dilemma. In the Diana example, our subject specifies some possible middle-way solutions that ought to be attempted prior to making a hard choice (e.g. subject's point 4 and 5).

In order to compare the practical complexity of the experienced and novice subjects' responses in terms of these cognitive operations, in our earlier study we classified our analyses of 212 protocols into three mutually exclusive categories of increasing sophistication of component use (Levels A, B and C; In the current study, we have augmented the definition of complexity as shown in Table II). We found that the graduate students clearly employ more complex and more contextually sensitive ethical strategies. In most of our high school subjects' protocols, students provided a simple action and justification for their resolutions (Component 3 and 4) 


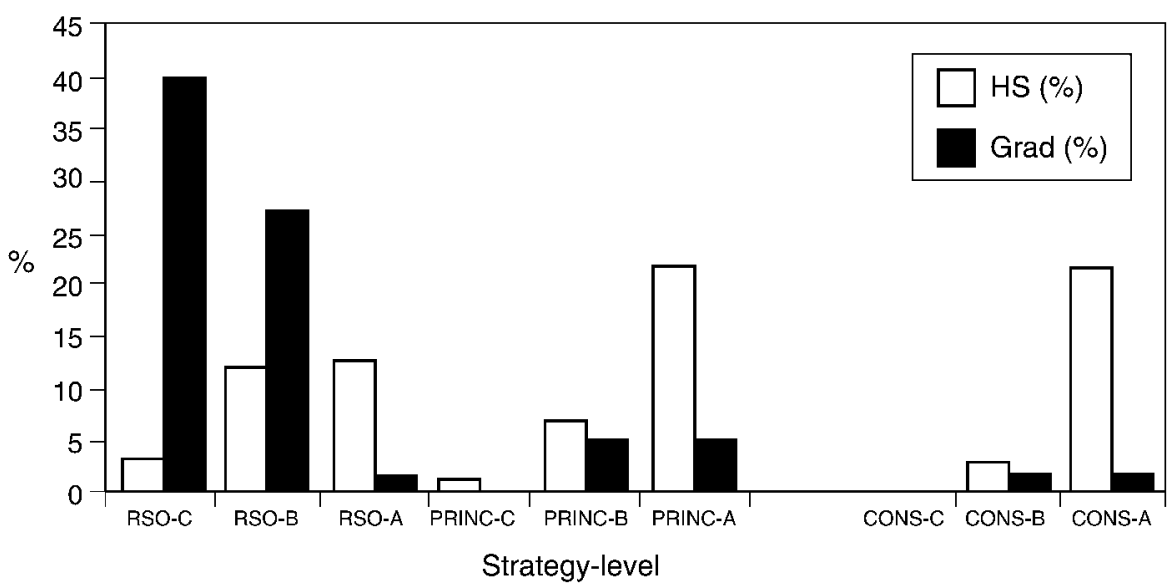

FIG 1. Level of strategy: high school vs. ethics grad. students.

(level A). Whereas, the graduate students were much more likely to also specify conditions under which specific professional role obligations recommend actions, and/or to consider their consequences in light of these conditions (Component 1 and 6) (level B); or, in addition, to appeal to hypothetical situations or extreme cases (Component 5) and/or generate creative middle way solutions to protect threatened values and honour relationships and obligations (Component 7) (level C).

Figure 1 shows the level of Component Sophistication employed by high school and ethics graduates as defined by the levels $\mathrm{A}$ to $\mathrm{C}$ defined above, and grouped according to the type of principle or justification applied-i.e. RSO; PRINC and CONS. The figure shows the total percentage of novice (high school) and experienced (medical ethics graduate student) responses evidencing any of the three strategy levels, A, B or C. Thus, Fig. 1 shows that only about $3 \%$ of the novice responses reached the $\mathrm{C}$ level (the most complex level). By contrast, slightly over $40 \%$ of the more experienced reasoners' responses reached the $\mathrm{C}$ level. The percentage of experienced reasoners' responses at simpler levels of complexity of the RSO strategy decreases monotonically (Ashley \& Keefer, 1996). There were also marked differences between the high school and graduate students' choice of ethical strategy. Of the 63 graduate student responses, $68 \%$ evidenced a choice of the RSO strategy (Level A, B or C). The 149 high school responses were spread out much more evenly across the different strategies, with $30 \%$ evidencing a choice of PRINC, $28 \%$ a choice of RSO, $24 \%$ CONS, and rest were coded as either non-moral, unelaborated (i.e. uncodable), and mixed. In this study we did not distinguish between high (MT) and middle (RSO, CONS, PRINC) level principles in Component 4 coding. The few higher level (MT) principles that (mostly graduate students) generated were coded as either PRINC (in the case of deontological principles) or CONS (in the case of utilitarian principles).

To summarise the results of this earlier study, we found that experienced ethical reasoners (i.e. the ethics graduate students) were careful to identify issues and to 
specify conditions under which specific professional role obligations recommend particular actions, that they elaborated conditions which would affect the moral analysis of a problem, in part through posing hypothetical variations of the problem, and that they justified resolutions in terms of those conditions which they conclude apply in the problem.

\section{Comparision of Ethicists and Undergraduate Engineering Students}

In the current study, we made use of an on-line corpus of engineering ethics cases, queries, and problem strategies, drawn from authoritative sources, and vetted by experienced professional judgement. Originally, we had intended to collect cases and commentaries from ethicists and professionals (in engineering) but we were pleased to discover that Michael Pritchard, Charles Harris and Michael Rabins had already assembled a fine set of cases and commentaries (some of which ultimately appeared in their textbook, Engineering Ethics (Harris et al., 1999)). These authors asked the ethicists and professionals in their study to write detailed commentaries on several ethical cases involving an engineering context. We were very fortunate when Michael Pritchard graciously made them available to us. Our goal was to use this corpus to investigate in detail how novice subjects interpret experienced reasoners' ethical analyses and whether novices can learn concepts and skills in a tutorial process which leads them through those analyses to help them reflect on their own processes of ethical decision-making. Another goal for this study was to develop a resource for ethical decision-making aimed at helping students identify moral issues and apply codes of ethics to ethical conflicts characteristic of their professional practice. Beyond this pedagogical goal, we expected our study would further understanding of some of the theoretical and epistemological issues surrounding fostering practical ethical decision-making. Our ultimate objective, which will be the subject of subsequent research, is to build an intelligent tutoring system that uses a similar but larger corpus of cases and analyses and that can facilitate novice reasoning in a highly interactive and principled way.

Our subjects for this experiment were undergraduate engineering students participating in a joint Engineering Programme at Washington University and the University of Missouri-St Louis. The experiment had three phases: pre-test, intervention, post-test. The pre-test and post-test were the same for all groups while the two interventions were different. In order to collect our data we created an experimental website, http:;drwww.pitt.edu/ estudy/ which presented students with: (1) a set of authentic engineering ethics cases, (2) suggestions on moral problem-solving based on the above model, (3) commentaries about the cases prepared by experienced ethicists and (4) metacommentaries, prepared by the first author that attempt to match the reasoning in the commentaries to the component reasoning model. The ethicists' commentaries were marked-up with hypertext links to relevant metacommentaries, which make clear how the linked portions of the commentaries relate to the suggestions on moral problem-solving (and thus to our model). For the purposes of this study we chose 10 different ethicists' responses to the first five cases (only). 
The website has five versions corresponding to our three experimental conditions, Group $3 N=8$, Group $2 N=8$ and Group $1 N=8$ (i.e. Experimental Group 2, Experimental Group 1 and Control Group for a total $N=24$ ), and our pre-test and post-test. In the experiment, subjects in Group 3 had access to all of the explanatory structure we have devised. Subjects in Group 2 and Group 1 saw successively more restrictive subsets of that information. In particular, the Group 3 version presented items (1) to (4). Version Group 2 presented only (1), (2) and a version of (3) without the mark-ups. Version Group 1 presented only (1) and (2).

In the pre-test, the students were presented with the facts of five cases. They were also presented with suggestions on moral problem-solving and were asked to prepare short written analyses for each case and answer a set of questions. The suggestions that we recommended matched each of the components of our model (i.e. Components 1-7 in Table I). Using the website, subjects emailed their answers to us. The post-test was written approximately 1-3 weeks later. It was nearly the same as the pre-test, except that five new cases were introduced. After the pre-test, the students were assigned at random to Group 3, Group 2 or Group 1. Their assignment for the week was to use the corresponding website version to work through the five pre-test cases in some detail, spending between 40 and 60 minutes per case to prepare answers to some additional intervention questions. Basically, Group 3 and Group 2 students used the website to read and respond to the views advanced in the ethicists' commentaries. The Group 1 students were asked to construct alternative views for themselves and then to respond to them.

\section{Analysis of Student Protocols}

Our protocol analysis applies methods developed in discourse analysis and extends the work of Ashley (1990), Keefer and Olsen (1995), Ashley and Keefer (1996) and Keefer et al., 2000). The components for the model are outlined in Table I. Relevant knowledge (Component 2) was analysed further into the six subcategories of Table IV.

The following is an example of a PRINC strategy using Component 2, 3 and 4 where the salient issue appears to be breaking a rule or guideline and the moral injunction to treat others fairly. The dilemma, On the Job, describes severe damage to a water filtration plant due to an open caustic valve in a seldom-used area of the facility. Carl, who is the plant supervisor, noticed on his first day of work that the safety system for the wastewater was different than for the acid system and was inadequate. Rick is the employee who leaves the valve open due to a lack of concentration stemming from fatigue brought on by studying for final exams.

In this case I think that Carl should report Rick as he is supposed to do in such situations. Nowhere does it say in the case that Rick will be terminated. Carl should follow the guidelines set by the management for such situations since, if he does not report Rick now and it happens again to someone else, should Carl treat him in the same manner as he treated Rick? Where is the line drawn? 
Following is an example of a CONS strategy using Component 1, 3 and 4 where the salient consideration appears to be assigning moral responsibility on the basis of the "effect" or consequences of the action.

I think it depends on the severity of the problem. If someone is going to get fired, might as well be the guy who screwed up. So what should Carl do? I think he should gauge the effects of having the caustic system open and see if it can be fixed. Secondly, if it can be fixed, he should have a major sit-down talk with Rick and maybe even chew him out. IF the problem is major, I do not think Carl really has a choice. Rick has got to go. It was his ignorance that cause the problem, he should pay.

The following is an example of an RSO strategy using Component 1, 2, 3 and 4 where the salient consideration appears to be the identification of moral issues by analysing the domain-specific features of the situation and justifying actions on the basis of role-specific obligations.

A more important problem is the lack of an alarm in this seldom used area. Carl noticed this situation the first day on the job. He asked why the two systems were so different, and the response should not have satisfied him. This incident occurred several months later, which implies that Carl did not fix the problem. Also, the tanks have vents piped to trench drains in the floor that are connected to the publicly owned wastewater treatment works. As an engineer, Carl should have noted the number of people affected by any negligence of the alarm system. Since the pipes were connected to a public water work, this should have been reason to match the acid and caustic safety systems. If Rick were required to hold the switch open while the tank was filling (as he would in the acid system), this event would have never happened. Therefore, I conclude that Rick is not to blame for the incident. He should not have been at work if he had not slept, but the incident is a direct result of Carl not mandating a safety system for the caustic system. He was aware of the possible problems and it never mentions any attempt on Carl's part to add these features.

To assist our coding the relevant known and unknown knowledge we first enumerated a list of the facts appearing in the cases prior to coding. Using the dilemmas as well as the ethicist's commentaries, each relevant knowledge component identified was further coded as specialised or common and, then, whether it was known (i.e. is given in the case description), unknown, (i.e. not given but important to the case), or finally, hypothetical or inferred. Hypothetical and inferred knowledge consisted of new knowledge generated by the respondent that could plausibly be inferred or deduced given the case description or scenario. The RSO protocol quoted above provides a good example of a student's identification of a good deal of relevant specialised knowledge in a case. Equally important as the relevant specialised professional knowledge needed to identify moral issues, is the ability to identify the relevant unknown facts that also bear on the identification of moral issues and assessment of moral responsibility. 
In the course of identifying the moral issues in another case, one of our ethicists illustrates the importance of considering relevant specialised known and unknown knowledge. This case describes a scenario where a manufacturer has made a commitment to supply component parts that it cannot keep. The head of quality control, Tim Vinson, must decide whether to switch to a less reliable part, not meet the deadline, or talk to the client.

No explanation is given of why the component is in short supply, or why Tim does not find this out until the 8 th, or whether he could or should have done something to assure that, short supply or not, his share of the components would arrive on time, except that he is said to "feel responsible" for not seeing the problem earlier. It is possible that he or someone has been negligent somewhere along the line; but it seems likely that the supplier is at fault for failing to deliver the components, which suggests that Ruskin has a lawsuit against the supplier and thus a means of shifting any penalty it suffers for failing to make timely delivery to Parker.

All protocols for each case $(N=240)$ were first coded for reasoning (e.g. Component $1,4,5,6,7$ ) and then for knowledge (Component 2). Next we coded the moral issues and justifications according to their content as set forth in Table III (i.e. for the PRINC strategy, a principle or norm, for the CONSEQ strategy, an appeal to consequences, for an RSO strategy, an appeal to role specific obligations, and for the MT strategy, an appeal to a moral theory. The minimal criteria for scoring a protocol as RSO, PRINC and CONSEQ was the presence of a simple action or plan (Component 3) followed by a justification (Component 4) or preceded by a moral issue (Component 1).

Of course, many subjects generated more than these minimal criteria for strategy identification. In order to provide a measure of the sophistication of the component use, we assigned each protocol to one of four increasing levels of complexity defined in Table II. Our results showed that the majority of the protocols had consistent content in the reasoning components (especially the students), which provides support for the idea that these strategies describe consistent or coherent approaches to moral conflict resolution. Protocols coded as having heterogeneous content were coded as mixed (including in the analysis below, mixed CONS and PRINC and mixed RSO and CONS/PRINC). Reliability measures were high to adequate for both strategy and component coding. Reliability for the component category coding was facilitated by first coding the knowledge content for each of the ten dilemmas before protocol analysis.

\section{Results}

Using the coding scheme referred to above; we have analysed the experienced ethicists' commentaries and the subjects' pre- and post-test written protocols in order to identify any differences in the use of the strategies, reasoning and knowledge component complexity as defined in our model of case-based ethical problem-solving. The percentage relationship between ethicists' and engineering 


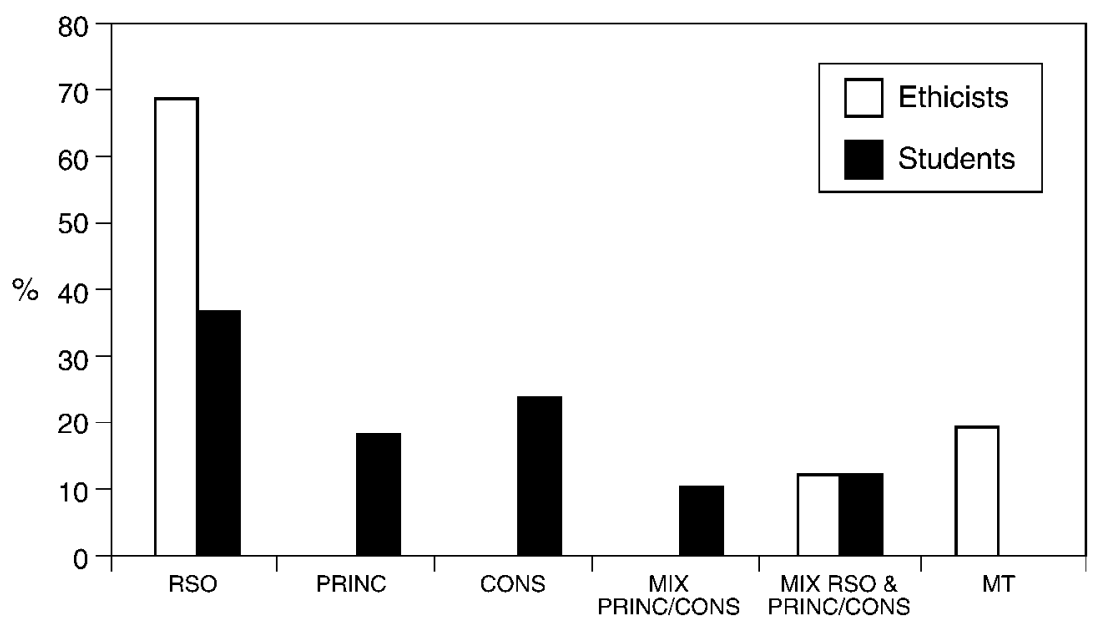

FIG 2. Ethicists' and engineering students' strategy choice.

undergraduates' choice of ethical strategies is shown in Fig. 2. As anticipated, the ethicists placed greater reliance on the RSO and the MT (moral theory) strategies whereas the undergraduates were far more likely to use each of the three strategies (including mixed CONS and PRINC and mixed RSO and CONS/PRINC). However, it is also important to note that even the ethicists used the MT strategy only sparingly. This result would lend support to those philosophers who question the importance of higher-level moral principles (based on moral theory) in resolving practical ethical conflicts (Jonsen \& Toulmin, 1990; Walzer, 1995).

Figure 3 shows a percentage comparison for the level of complexity in component use for ethicists' and students' responses, as defined above. Again, as we expected, the ethicists tended to use more of the components when resolving a particular dilemma (e.g. level D) than did the undergraduates, whose responses were most likely to be at the $\mathrm{B}$ level of complexity. The contrast is quite dramatic, as the mean number of components used in the RSO, PRINC, and CONSEQ undergraduate protocols was 2.2 per case while the mean number of components used in the ethicists' protocols was three times that amount at 6.07 . These data suggest that the ethicists are much more able to consider moral issues from a variety of perspectives, and are more likely to consider alternative resolutions.

Figure 4 shows a percentage comparison for the students' and ethicists' use of specialized vs. common knowledge. As well as considering additional moral issues and alternate views, the ethicists also identified considerably more contextual knowledge they determined to be relevant to the case. For example, the ethicists' generated an average of 5.57 knowledge components per case resolution $(N=14)$ compared to 1.46 per case for the undergraduates $(N=233)$. Perhaps more importantly, this difference was proportionally much larger for specialised as compared with common knowledge with 4.64 SK to $0.93 \mathrm{CK}$ knowledge components per case for the ethicists and $0.95 \mathrm{SK}$ to $0.52 \mathrm{CK}$ for the students. The ethicists also appear 


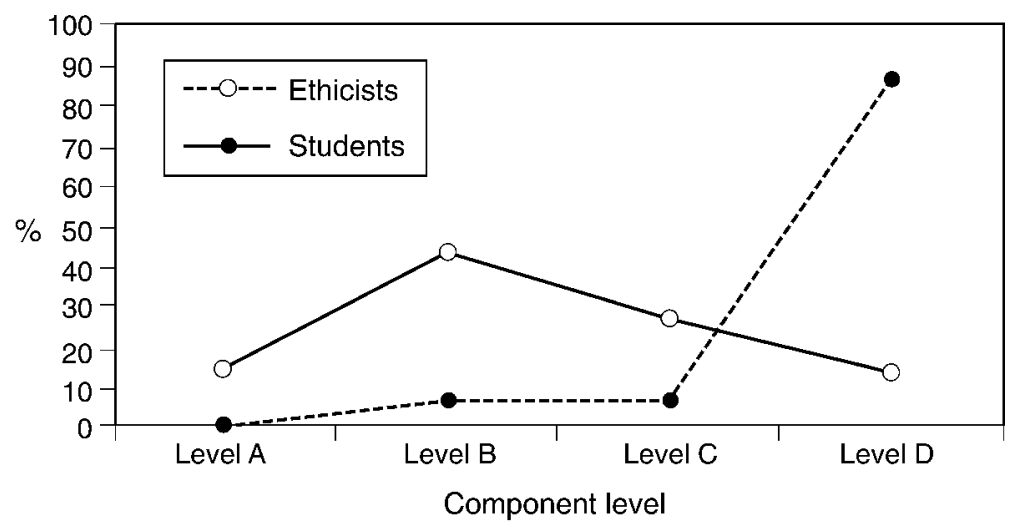

FIG 3. Students' and ethicists' component complexity.

to be far more likely to generate relevant unknown specialised knowledge in their responses than students. The ethicists' identified 17 relevant unknown facts (SK) in 14 responses, whereas the students only identified 12 in a total of 223 case responses. Finally, Fig. 4 shows some striking differences in the use of knowledge by type for both the ethicists and the students. As the figure indicates, the ethicists generated considerably more hypothetical and inferred knowledge components when discussing specialised knowledge than did the students. While the students would recall salient professional knowledge identified in the case, the ethicists were more likely to reason with that knowledge in crafting their resolutions, i.e. using that knowledge in order to make reasonable inferences, consider hypothetical situations, etc.

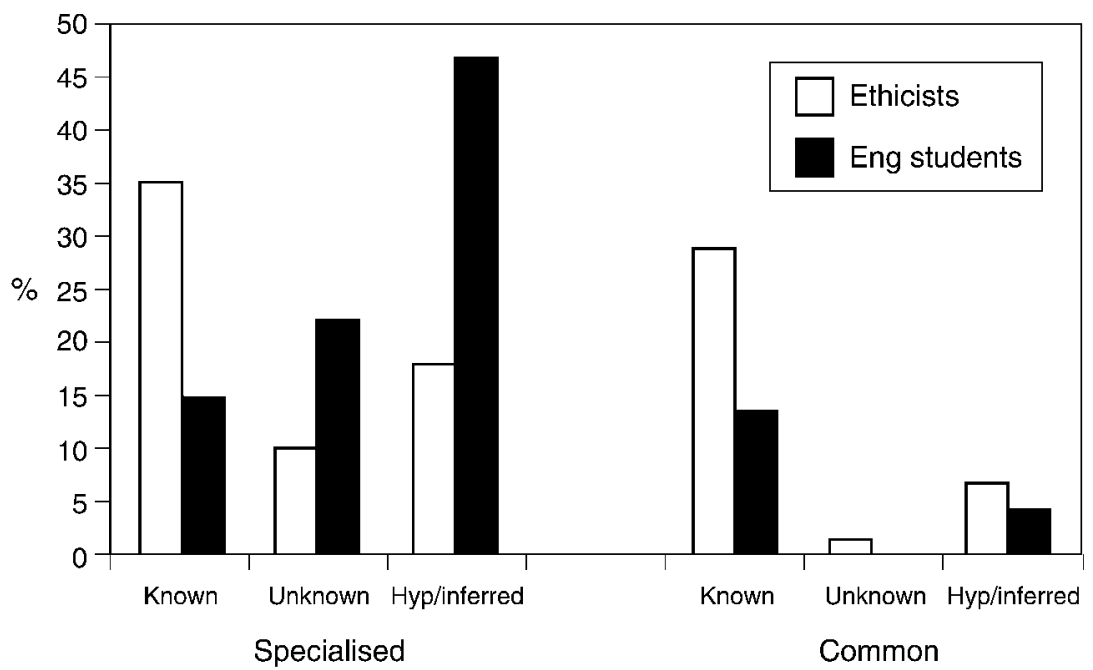

FIG 4. Knowledge type comparisons. 


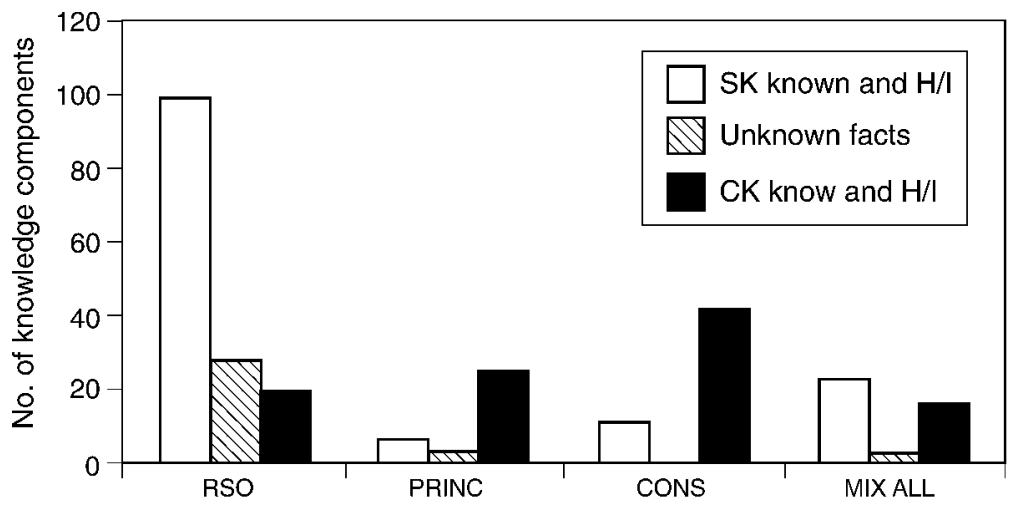

FIG 5. Knowledge type by strategy.

Figure 5 compares the number and type of specialised, common and unknown knowledge components identified by students using the RSO, PRINC and the CONS strategies. As Fig. 5 shows, students identifying RSO issues and applying RSO principles were more likely to identify relevant knowledge in the dilemmas and also apply more specialised (SK) than common (CK) knowledge. In contrast, students using the PRINC and CONS strategy identified comparatively less specialised knowledge and relatively more common everyday knowledge with the CONS student protocols exhibiting the largest amount of common knowledge use. This difference suggests that attending to specialised knowledge is probably more important in aiding those students using the RSO strategy to determine the salient professional ethical obligations in the case. RSO protocols were also far more likely to identify the morally relevant unknown facts (UF) that are not identified in the dilemma but are important to its analysis and resolution.

In regard to moral problem-solving component and strategy interactions, the RSO student protocols also contained more components per case than the CONS and PRINC (mean of 3.01-2.75, respectively). This is in line with our results in the previous study where we found that students using the RSO strategy were more likely to use more components in their responses. However, more striking than this comparatively small overall difference is the variance in the type of component use for the different strategies. Figure 6 compares the number of reasoning components applied for the RSO professional ethics strategy with those using the CONS and PRINC strategies. As the figure shows, the CONS and PRINC strategies exhibit relatively more use of Component 1 and 4, while the students using the RSO strategies were more likely to use the reasoning components associated with casebased approaches (e.g. Component 5, 6 and 7). These data would support the view that consequence and principle-orientated approaches to ethical decision-making place greater emphasis on the justification of moral actions in practical conflicts. For example, the CONS strategy protocols exhibited nearly three times as many moral justification components (i.e. Component 4) than were present in the RSO strategy protocols. 


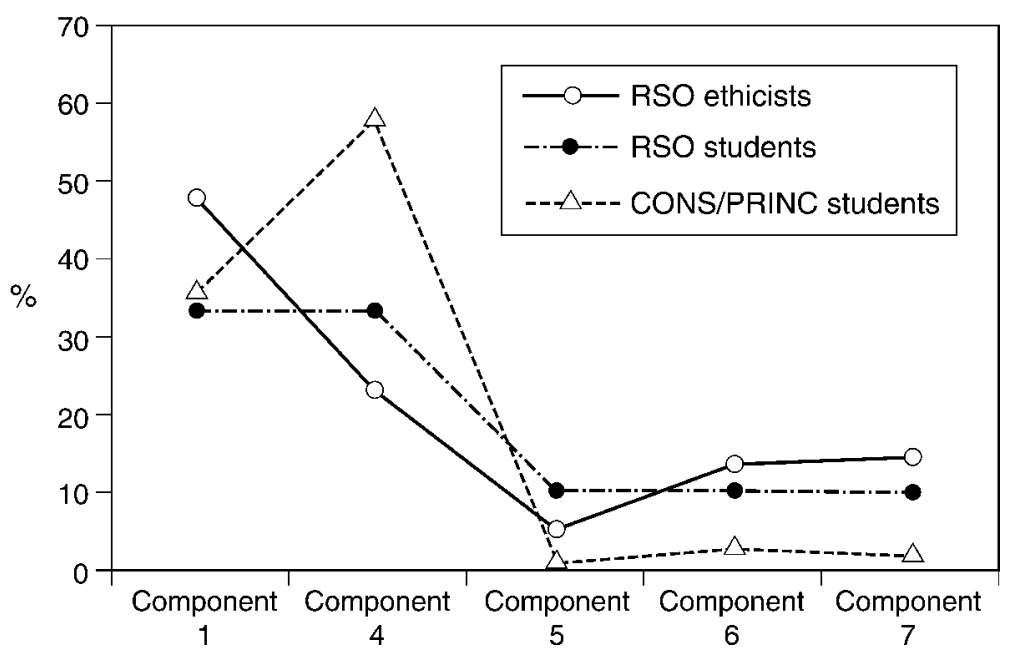

FIG 6. Reasoning component use by strategy.

With respect to our intervention, we hoped that after several cases the Group 3 subjects would be better able than Group 2 or Group 1 subjects to appreciate the RSO strategy and use of the specialised knowledge that the ethicists employ and apply them spontaneously to new cases. Similarly, we hoped that the Group 2 subjects would be better in this respect than the Group 1 subjects. While the pre- and post-test differences were in the right direction they were small and non-significant in regard to both increased component and knowledge use. The gains in the use of SK pre- to post-test were greater for both experimental groups but the differences were small. In regard to the increase of RSO strategy use, there were modest gains for both experimental groups while the control group's use of the RSO strategy remained constant. We also have some qualitative evidence that the students found the dialogue with the ethicists engaging, and found the comments and mark-ups useful. However, due to the small sample size and the fact that there was some variation in the groups' baseline choice of strategies it is difficult to interpret the effect of this brief intervention.

Finally, we also noticed that students tended to favour use of a particular strategy in their pattern of responses (see Fig. 2). However, we expect that most students have understanding of and are able to employ each of these strategies. There is also evidence that there are other factors besides individual preference that affect the choice of strategy. For example, in line with psychological research on moral reasoning (Johnson, 1988; Keefer \& Olsen, 1995) we observed that different types of dilemmas tend to "pull" for different components and even different content strategies. 


\section{Discussion}

In comparing the protocols of arguments about practical ethical dilemmas by undergraduate engineering students and more experienced ethicists in this study, we have observed differences in the complexity of component use, the choice of strategy and, we believe, the overall quality of responses. While we would not claim that any singular ethicists' response can be considered better in quality than another ethicist's or student's response, we do believe that, as a group, the ethicists' protocols can be judged superior in many respects. The experienced ethicists' responses clearly favoured the RSO strategy and they used far more moral problem-solving components (reasoning and knowledge) in their resolutions. In terms of reasoning, our data show that the ethicists were more likely to identify different moral issues, consider alternative scenarios that argue for different conclusions, offer alternative resolutions that take into account divergent moral claims, and identify and evaluate moral consequences of their responses than were the students.

Perhaps more importantly our data show that, as a group, protocols making use of professional knowledge and knowledge of professional obligations appear to exhibit better-quality reasoning than protocols employing a more justificatory emphasis. As noted above, there were strong differences in the relative distribution of component use by strategy with RSO using more of Component 5, 6, 7 and PRINC/CONS using more Component 4 (justification-see Fig. 6). This result is in line with our previous finding that the PRINC/CONS strategy attends more to the justificatory properties of moral problems (Keefer \& Olsen, 1995; Ashley and Keefer, 1996; Keefer et al., 1998). Protocols of students using a justificatory approach appear more likely to recommend simple actions or simple plans as solutions and to adduce rules, principles, or simple consequences in their defence.

We believe this finding is important as it points to the limitations of philosophical and psychological analyses that, by focusing exclusively on the justification for moral decisions, have minimised the more deliberative or practical dimensions of ethical reasoning. Indeed, in response to some traditionally more procedural or principled approaches of modern ethical theorising, many philosophers have argued that moral philosophy needs to recover a more agent-centred and deliberative understanding of ethical thinking (Hampshire, 1983; Taylor, 1989; MacIntyre, 1984-see also Keefer, 1996; Nussbaum, 1986). For example, Hampshire (1983) argues that modern procedural approaches have placed too much attention on the justification of moral actions, including the acquisition of moral principles, and the use of rules or principles in providing the standard for moral judgement. The practical effect of this tendency has been to conceive moral problems essentially from the perspective of the moral "judge" or "critic" while ignoring the deliberative perspective of the moral "actor" or "agent".

The theoretical and empirical work of Piaget (1932/1965) and Kohlberg (1984) has generally supported this trend, both methodologically and theoretically. The theory describes moral judgement as passing through a series of stages culminating in the application of high order general principles to practical judgements. Methodologically, after presentation of one of the several dilemmas in Kohlberg's Moral 
Judgment Interview, the format requires subjects to first respond to a yes or no question to determine whether the protagonist ought (or ought not) to perform a simple action or plan (e.g. "Should Heinz steal the drug"). Subsequent to that response, subjects are then asked specifically to justify their choice. It is the arguments that respondents provide to this (justificatory) question that are then analysed and scored for stage reasoning [1].

Our intention is not to minimise the importance of the quality of the ethical justification in case-based responses, but to alert us to the problem of equating the moral reasoning component of the ethical response with moral justification. In Rest's (1983) model four components are enumerated and deemed essential to understanding moral conflicts. Yet, as Rest follows Kohlberg in equating moral reasoning to moral judgement, it can be argued that the reasoning component of this model may also be conceived too narrowly from within a justificatory framework. In contrast, our own model posits several reasoning components in addition to justification as integral to mature ethical decision-making. These include the identification of moral issues, the use of relevant known and unknown professional knowledge, the consideration of alternate scenarios identifying alternate issues and actions, the search for creative alternatives, and attention to the long-term moral and practical consequences of actions.

Given this complexity, there is a valid concern that a focus on ethical justification can have practical "costs". Our fear is that if ethical problem-solving is conceived primarily as the attempt to find the best justification for choosing one action over another in a conflict, this can produce an "exclusionary" mind-set that might, in turn, short change the search for creative alternatives. For example, the student's response to the On the Job case (quoted above) indicates how, in the exercise of the PRINC Strategy, determining that one principle is of overriding importance may invoke this mind-set perhaps eliminating consideration of conflicting reasons, knowledge issues and alternative resolutions. In the example, Rick's negligence and the need for Carl to treat all employees the same may play a role in the failure to consider other more systemic issues pertaining to safety and corporate accountability. We believe that any factors that can reinforce the presumption that a case conflict is irreconcilable are unfortunate since, as Whitbeck argues, "it defeats any attempt to do what design engineers often do so well, namely, to satisfy potentially conflicting considerations simultaneously" (Whitbeck, 1998, p. 56).

Finally, to claim that the RSO protocols were superior is not to lay claim that professional morality is somehow superior to common morality, just as it would be foolish to claim that professionals are somehow, as a group, more moral than non-professionals. On the contrary, it is our belief that role-specified "middle-level" principles of professional morality are dependent and derive from the "middle-level" principles of common morality. Professional ethics codes attempt to apply and extend common morality to the special obligations that derive from specialised professional knowledge. Similarly, recognising the importance of professional knowledge and their associated role-specific principles does not commit one to any argument for stronger autonomy in the professions. Professional ethics codes can 
uphold but also undermine the strictures of common morality. For example, some earlier versions of ethics codes had provisions dedicated only to preserving the autonomy and status of the profession (e.g. strictures against advertising, etc.). Other provisions were more blatantly self-serving. For example, earlier versions of medical and engineering codes contained provisions that banned criticising the work of fellow practitioners (Whitbeck, 1998).

Similarly, the principles that attach to professional roles should never be considered immutable or non-revisable. Just as personal moral judgements need to be tested and refined by appeal to experience, the value of professional codes (and their role specific ethical principles) need to be tested by their ability to apply successfully to cases. Indeed, the NSPE codes have changed and added provisions based on challenges that have arisen in the practical arena (Whitbeck, 1998). In addition, many codes of ethics rely on a somewhat outdated view of professionals as self-employed custodians of specialised knowledge, whereas most professionals will practice their trade in larger corporate and social contexts that provide additional ethical responsibilities and challenges (May, 1996).

The results of our empirical investigations support the position of those who argue for the pedagogical importance of case-based approaches to teaching professional ethics. Given the challenges young professionals face in interpreting the open-ended quality of professional codes and, given the ever-changing character of the professions themselves, the importance of understanding code principles in the context of cases is clear. In addition, case-based approaches may have a role to play in broadening our view of the components that are necessary for teaching professional ethics effectively. In the language of early research in the cognitive sciences, case-based problems are, for the most part, "ill-structured". Practical ethical dilemmas appear in contexts that are ripe with ambiguities, usually including inadequate or at least incomplete knowledge [2]. As we have shown above, there are several ways in which fully mature moral reasoning in a professional context can exhibit sensitivity to the diversity of moral claims that attend these moral conflicts. Cases that reflect such moral complexities provide relevant and more realistic challenges for young professionals. While complex case conflicts do offer serious moral constraints on possible courses of actions, they rarely yield themselves to one unique correct moral solution. This suggests an essential additional ingredient of ethical instruction may be to include opportunities for student dialogue, disagreement and discussion. Authentic cases are particularly well suited for stimulating just such productive discussions and dialogue.

Case-based ethical reasoning in applied professional contexts provides exciting opportunities and challenges not only to students, ethicists and professionals, but also to those who wish to analyse and understand it theoretically. Acquiring a specialised body of knowledge, especially knowledge that will affect the well-being of others, is perhaps the defining feature of a being and becoming a professional. According to Whitbeck, "[b] ecause society looks to members of a given profession to master and develop knowledge in a particular area, the members of a profession bear special moral responsibilities in the use of the special knowledge vested in them" (Whitbeck, 1998, p.40). Our analysis of the mature case-based reasoning that 
ethicists and certain students apply in professional contexts suggests that attention to that knowledge, and how that knowledge relates to the identification of role-related moral principles, may be an essential part of professional ethical instruction.

\section{Acknowledgements}

This research was funded by the National Science Foundation's Ethics and Values Program NSF SBR-9714411. The authors would like to thank Julian Osuji for his assistance in data collection for this study.

Correspondence: Dr Matthew Keefer, University of Missouri-St Louis, Division of Educational Psychology, 8001 Natural Bridge Road, St Louis, MO 63121, USA; Tel: (314) 516 6081; Fax: (314) 516 5784; E-mail: keefer@umsl.edu

Dr Kevin D. Ashley, University of Pittsburgh, Learning Research and Development Center, 3939 O’Hara Street, Pittsburgh, PA 15213, USA; Tel: (412) 624 7496; Fax: (412) 6249149.

\section{NOTES}

[1] In contrast, the scheme that Carol Gilligan preferred encouraged respondents to reply to the more open-ended pract question of "What should the protagonist do?" Subsequent research has shown that the kind of resolutions respondents produce is influenced by whether the question "pulls" for a justificatory or a deliberative (i.e. practical) approach. (Johnson, 1988; Keefer \& Olsen, 1995). Applying a cognitive discourse analysis to respondents' dilemma responses Keefer and Olsen (1995) found that in certain conditions different respondents will produce either more deliberative or more justificatory resolutions. The justificatory protocols would provide a simple action or plan followed by an appeal to a general principle or consequence, while the deliberative protocols identified moral issues in a narrative using interactive planning sequences that provided the context for (what in this paper we term) alternative resolutions. In addition, each protocol was also independently coded for either "justice" or "care" orientation. Interestingly, the justificatory protocols were strongly associated with "justice" orientation, while deliberative or practical protocols were strongly associated with the "care" orientation.

[2] In other work by the second author, an Artificial Intelligence (AI) computational model was used to demonstrate empirically that, as the NSPE Board of Ethical Review decides and explains cases, it fleshes out or "operationalises" the meaning of the abstract ethics code provisions. In an objective experiment, it was shown that the program, called sIRocco (i.e. System for Intelligent Retrieval of Operationalised Cases and Codes), retrieves relevant cases and code provisions more effectively with the accumulated operationalisation information than without it (Ashley \& McLaren, 2001).

\section{REFERENCES}

ARRAS, J.D. (1991) Getting down to cases: the revival of casuistry in bioethics, fournal of Medicine and Philosophy, 16, pp. 29-51.

AsHLey, K.D. (1990) Modeling Legal Argument: reasoning with cases and hypotheticals (MIT Cambridge, Press).

AshlEy, K.D. \& KeEFer, M.W. (1996) Ethical reasoning strategies and their relation to case-based instruction: some preliminary results, Proceedings of the Eighteenth Annual Conference of the Cognitive Science Society, pp. 483-488. 
Ashley, K.D. \& MCLAREN, B.M. (2001) An AI investigation of citation's epistemological role, in: Proceedings of the Eighth International Conference on Artificial Intelligence $\mathcal{E}$ Law (ICAIL-01), pp. 32-41 (Association for Computing Machinery, New York).

Beauchamp, T. \& Childress, J.F. (2001) Principles of Biomedical Ethics, 5th edn (New York, Oxford University Press).

GrIFFIN, N. (1988) Using "Ethics in Teaching" in teaching in ethics: student views on the case studies, Teaching Philosophy, 11, pp. 116-127.

HAMPshire, S. (1983) Fallacies in moral reasoning, in: A. MACInTYre \& S. HAUERwas (Eds) Changing Perspectives in Moral Philosophy (Notre Dame IN, Notre Dame University Press).

Harris, C., Pritchard, M. \& Rabins, M. (1999) Engineering Ethics, 2nd edn (Belmont, CA, Wadsworth).

Johnson, K. (1988) Adolescents' solutions to dilemmas in fables: two moral orientations-two problem solving strategies, in: C. Gilligan, J. Ward, J. TaYlor \& B. Bardige (Eds) Mapping the Moral Domain, pp. 49-71 (Cambridge, MA, Harvard University Press).

Jonsen, A.R. (1991) Casuistry as methodology in clinical ethics, Theoretical Medicine, 12, pp. 295-307.

Jonsen A.R. \& Toulmin S. (1990) The Abuse of Casuistry: a history of moral reasoning (Berkeley, CA, University of CA Press).

KEEFER, M.W. (1996) The inseparability of morality and personal well being: the duty/virtue debate in moral education-revisited, fournal of Moral Education, 25, pp. 277-290.

KeEFER, M.W., Ashley, K.D. \& OsujI, J. (1998) Case-based ethical instruction: providing a context for student dialogue with experienced ethicists, Paper presented at the Association for Moral Education Annual Meeting, Dartmouth College November 19-21.

KEEFER, M.W. \& Olson, D. (1995) Moral reasoning and moral concern: an alternative to Gilligan's gender based hypothesis, Canadian fournal of Behavioral Sciences, 27, pp. 420-437.

KeEFER, M.W., ZeITZ, C.M. \& ReSNick, L.B. (2000) Judging the quality of peer-led student dialogues, Cognition and Instruction, 18, pp. 55-83.

KohlBerg, L. (1984) The psychology of moral development: the nature and validity of moral stages, in: L. Kohlberg (Ed.) Essays on Moral Development, Vol. 2. The philosophy of moral development (San Francisco, CA, Harper \& Row).

LAVE, J. \& Wenger, E. (Eds) (1991) Situated Learning: legitimate peripheral participation (New York, Cambridge University Press).

MacIntyre, A. (1984) After Virtue, 2nd edn (Notre Dame, IN, University of Notre Dame Press).

MAY, L. (1996) The Socially Responsive Self: social theory and professional ethics (Chicago, University of Chicago Press).

Nussbaum, M. (1986) The Fragility of Goodness: luck and ethics in Greek tragedy and philosophy (Cambridge, Cambridge University Press).

Piaget, J. (1965) The Moral fudgment of the Child (New York, NY, Free Press) (original work published 1932).

RESNICK, L.B. \& WIRT, J.G. (Eds) (1996) Linking School and Work: roles for standards and assessment (San Francisco, Jossey-Bass Publishers).

Rest, J. (1983) Morality, in: P.H. Mussen (Series Ed.), J. Flavell \& E. Markman, (Vol. Eds) Handbook of Child Psychology, Vol. 3. Cognitive Development, 4th edn, pp. 556-629 (New York: Russell).

Strong, C. (1988) Justification in ethics, in: B.A. Brody (Ed.) Moral Theory and Moral Fudgments in Medical Ethics, pp. 193-211 (Dordrecht, Kluwer Academic Publishers).

TAYLOR, C. (1989) Sources of the Self (Cambridge, MA, Harvard University Press).

WALzER, M. (1995) Thick and Thin: moral argument at home and abroad (Notre Dame, IN, University of Notre Dame Press).

Whitbeck, C. (1998) Ethics in Engineering Practice and Research (Cambridge, Cambridge University Press). 